\title{
Prognostic impact of invasive haemodynamic measurements in combination with clinical and echocardiographic characteristics on two-year clinical outcomes of patients undergoing transcatheter aortic valve implantation
}

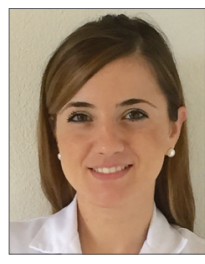

Anna Franzone ${ }^{1}$, MD; Crochan J. O’Sullivan ${ }^{1,2}, \mathrm{MD}, \mathrm{PhD}$; Stefan Stortecky ${ }^{1}, \mathrm{MD}$; Dik Heg ${ }^{3}, \mathrm{PhD}$; Jonas Lanz ${ }^{1}$, MD; René Vollenbroich ${ }^{1}$, MD, MPP; Fabien Praz ${ }^{1}$, MD; Raffaele Piccolo ${ }^{1}, \mathrm{MD}$; Masahiko Asami ${ }^{1}$, MD; Eva Roost ${ }^{4}$, MD; Lorenz Räber ${ }^{1}, \mathrm{MD}, \mathrm{PhD}$; Marco Valgimigli ${ }^{1}, \mathrm{MD}, \mathrm{PhD}$; Stephan Windecker ${ }^{1}, \mathrm{MD}$; Thomas Pilgrim ${ }^{1 *}, \mathrm{MD}$

1. Department of Cardiology, Swiss Cardiovascular Center, University Hospital Bern, Bern, Switzerland; 2. Department of Cardiology, Stadtspital Triemli, Zürich, Switzerland; 3. Institute of Social and Preventive Medicine and Clinical Trials Unit, University Hospital Bern, Bern, Switzerland; 4. Department of Cardiovascular Surgery, Swiss Cardiovascular Center, University Hospital Bern, Bern, Switzerland

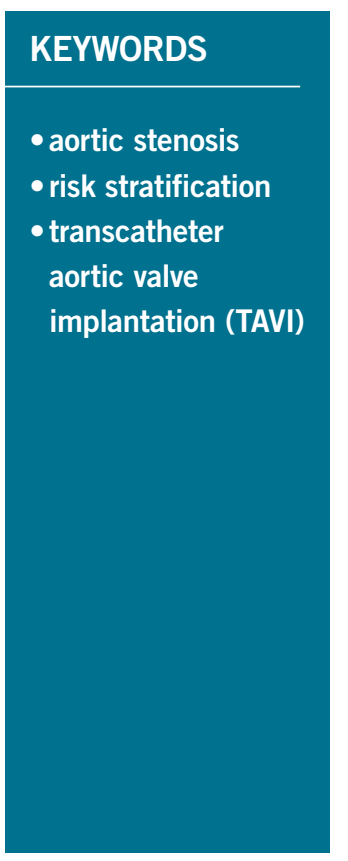

\begin{abstract}
Aims: The aim of the study was to evaluate the prognostic utility of right heart catheterisation (RHC)derived measures among patients undergoing transcatheter aortic valve implantation (TAVI).

Methods and results: Data of 469 patients included in the Bern TAVI Registry between August 2007 and December 2012 and undergoing preoperative RHC were analysed. The relationship between haemodynamic parameters and survival was evaluated with Cox proportional hazards models. At two-year follow-up, 118 patients had died (25.1\%). At multivariable analysis, diabetes (hazard ratio [HR] 1.95, 95\% confidence interval $[\mathrm{CI}]: 1.28-2.96, \mathrm{p}=0.001$ ), transapical access (HR 1.66, 95\% CI: 1.07-2.56, $\mathrm{p}=0.02$ ), and moderate or severe mitral regurgitation (HR $1.55,95 \%$ CI: $1.00-2.39, \mathrm{p}=0.04$ ) were independent predictors of two-year mortality, whereas no correlation between RHC-derived measures and mortality was found. Furthermore, the addition of haemodynamic variables did not significantly improve the prognostic power of a model incorporating clinical and echocardiographic data (Harrell's C-index: 0.667, 95\% CI: 0.615-0.719 vs. $0.662,95 \%$ CI: $0.612-0.713, \mathrm{p}=0.47)$.
\end{abstract}

Conclusions: On the basis of a comprehensive clinical and echocardiographic evaluation, RHC performed prior to TAVI does not add incremental prognostic value.

\footnotetext{
*Corresponding author: Department of Cardiology, Swiss Cardiovascular Center, Bern University Hospital, CH-3010 Bern, Switzerland.E-mail: thomas.pilgrim@insel.ch
} 


\section{Abbreviations}

AS

AUC

CI

HR

LVEF

NYHA

PAP

PARTNER Placement of Aortic Transcatheter Valves

RAP right atrial pressure

RHC right heart catheterisation

SD standard deviation

STS Society of Thoracic Surgeons

TAVI transcatheter aortic valve implantation

\section{Introduction}

Haemodynamic parameters measured at right heart catheterisation (RHC), such as mean right atrial pressure and pulmonary arterial compliance, have proved to be reliable predictors of outcomes in several clinical settings including pulmonary arterial hypertension and chronic heart failure ${ }^{1,2}$. Severe aortic valve stenosis (AS) produces significant changes in the interdependent left and right ventricular haemodynamics ${ }^{3}$. Indeed, sustained pressure overload induces the development of myocardial hypertrophy which progressively impairs the diastolic properties of the left ventricle; its heightened filling pressures are then passively transmitted backwards, eliciting a reactive component within the pulmonary vasculature ${ }^{4}$. Multiple lines of evidence support the negative impact of baseline pulmonary hypertension on clinical outcomes after transcatheter aortic valve implantation (TAVI) ${ }^{5-7}$. However, so far, a systematic appraisal of the predictive value of other RHC-derived parameters in this context is lacking. Our aim was to identify, among haemodynamic indices obtained at RHC, the determinants of mortality at two years after TAVI and to assess whether preoperative RHC could improve the risk stratification of patients undergoing TAVI.

\section{Methods}

\section{STUDY POPULATION AND ENDPOINT}

We analysed data of patients undergoing RHC prior to TAVI who were consecutively included in the Bern TAVI Registry between August 2007 and December 2012. TAVI was performed on the basis of a multidisciplinary consensus and as previously described $^{8}$. Active follow-up was planned at 30 days, one and two years by in-hospital visits or phone interview. All potential adverse events were adjudicated by a dedicated clinical events committee according to the standardised endpoint definitions proposed by the Valve Academic Research Consortium. The study adheres to the ethical principles of the Declaration of Helsinki. The Registry was approved by the local ethics committee, and written informed consent was mandatory for patients to participate. The primary study endpoint was all-cause mortality at two years.

\section{RIGHT HEART CATHETERISATION}

RHC was performed at the time of preprocedural coronary angiography, as part of a standardised institutional protocol for the comprehensive assessment of the severity of the AS and the global patient haemodynamic profile. Briefly, fluid-filled catheters connected to pressure transducers were used to record pressures. After review of haemodynamic traces, the following parameters were collected: mean right atrial pressures (mean RAP), systolic and end-diastolic pressures of the right and left ventricle and systolic, diastolic and mean pulmonary artery pressures (PAP). Cardiac output was computed with the modified Fick method and indexed to body surface area to obtain the cardiac index.

\section{STATISTICAL ANALYSIS}

Normally distributed variables were expressed as mean \pm standard deviation (SD), categorical variables as number and percentages. Multiple imputation of missing values using chained equations was used throughout to account for, and impute, missing data in predictor variables (20 data sets created), and Rubin's rule was used to combine the results of the data sets in all predictor analyses. Cox proportional hazards models using univariate analysis were used to test the association between predictor variables and mortality at two years after TAVI. Considering the large number of continuous predictor variables, each was additionally evaluated for potential non-linear effects (e.g., curvilinear or quadratic effects), but none was found. Estimates of hazard ratios (HR) and 95\% confidence intervals (CI) are provided. To investigate the prognostic contribution of RHC-derived parameters to clinical and echocardiographic findings at baseline, a stepwise procedure was used. Harrell's C-index and the area under the curve (AUC) for clinical predictor variables (age, body mass index, diabetes, previous cerebrovascular accident, chronic obstructive pulmonary disease, transapical access and STS score) were first determined (clinical model). Subsequently, we added the echocardiographic predictor variables (moderate or severe mitral regurgitation, left ventricular ejection fraction and mean aortic valve gradient) to the clinical model (clinical with echo model). Variable selection of these two models was based on previously published literature tagging these variables as relevant for mortality or risk assessment of TAVI patients, and therefore they were used irrespective of the effect on mortality in our patient population. Finally, the RHC-derived predictor variables were added singly, of which pulmonary arterial compliance and mean RAP were retained. Variable selection of the RHC-derived predictor variables was based on single inclusion into the clinical with echo model, and RHC-derived predictor variables were retained showing a $\mathrm{p}<0.3$ effect on mortality. The prognostic contribution of RHC was determined by comparison of AUC calculated at each step. All analyses were performed using Stata Statistical Software, Release 14 (StataCorp LP, College Station, TX, USA), and statistical significance was defined as $\mathrm{p}<0.05$.

\section{Results}

Of 606 patients included in the Bern TAVI Registry, 469 (77.3\%) underwent RHC prior to TAVI. The majority of TAVI procedures 
were performed through the transfemoral access (80\%); $18 \%$ of cases were transapical and $1 \%$ trans-subclavian. At two years, 118 patients $(25.1 \%)$ had died and clinical follow-up was available in 346 of 351 patients (98.5\%) still alive. Clinical, echocardiographic, and procedural characteristics were similar between patients who underwent RHC and those who did not (data not shown).

\section{BASELINE CLINICAL AND ECHOCARDIOGRAPHIC CHARACTERISTICS}

Table 1 shows the baseline clinical features of the entire population and of patients dead or alive at two-year follow-up. Compared to patients alive at two years after TAVI, patients who had died were found to have higher rates of diabetes mellitus (39\% vs. $25 \%$, HR 1.74, 95\% CI: 1.20-2.52, $\mathrm{p}=0.003)$, atrial fibrillation (71\% vs. $68 \%$, HR 1.46, 95\% CI: 1.01-2.11, $\mathrm{p}=0.04)$, and a greater operative risk (logistic EuroSCORE: $27.6 \pm 15.5$ vs. $22.4 \pm 13.1$, HR 1.02, $95 \%$ CI: $1.01-1.03, \mathrm{p}<0.001$ and STS score: $8.4 \pm 5.4$ vs. $6.4 \pm 4.1$, HR 1.06, 95\% CI: 1.04-1.09, $\mathrm{p}<0.001)$.

Table 2 shows echocardiographic parameters measured prior to TAVI. In the total cohort, mean left ventricular ejection fraction (LVEF) was $53.9 \pm 14.4 \%$ and mean aortic valve area (AVA) was $0.7 \pm 0.2 \mathrm{~cm}^{2}$. In univariable analysis, LVEF $(50.4 \pm 15.6 \%$ vs.

Table 1. Baseline clinical characteristics.

\begin{tabular}{|c|c|c|c|c|c|}
\hline & $\begin{array}{l}\text { Overall } \\
n=469\end{array}$ & $\begin{array}{l}\text { Patients alive } \\
\text { at } 2 \text { years } \\
n=351\end{array}$ & $\begin{array}{l}\text { Patients dead } \\
\text { at } 2 \text { years } \\
n=118\end{array}$ & $\begin{array}{c}\text { Hazard ratio } \\
\text { (95\% CI) }\end{array}$ & $p$-value \\
\hline Age (years) & $82.3 \pm 5.5$ & $82.2 \pm 5.5$ & $82.7 \pm 5.4$ & $1.01(0.98-1.05)$ & 0.41 \\
\hline Female gender, $\mathrm{n}(\%)$ & $264(56 \%)$ & $204(58 \%)$ & $60(51 \%)$ & $0.78(0.54-1.12)$ & 0.18 \\
\hline Height $(\mathrm{cm})$ & $164.7 \pm 8.2$ & $164.6 \pm 8.1$ & $164.9 \pm 8.6$ & $1.00(0.98-1.03)$ & 0.74 \\
\hline Weight (kg) & $71.3 \pm 15.2$ & $71.6 \pm 14.6$ & $70.2 \pm 16.7$ & $0.99(0.98-1.01)$ & 0.29 \\
\hline Body mass index $\left(\mathrm{kg} / \mathrm{m}^{2}\right)$ & $26.2 \pm 5.1$ & $26.4 \pm 5.0$ & $25.7 \pm 5.5$ & $0.97(0.94-1.01)$ & 0.15 \\
\hline Body surface area $\left(\mathrm{m}^{2}\right)$ & $1.8 \pm 0.2$ & $1.8 \pm 0.2$ & $1.8 \pm 0.2$ & $0.64(0.28-1.47)$ & 0.30 \\
\hline Diabetes mellitus, n (\%) & $133(28 \%)$ & $87(25 \%)$ & $46(39 \%)$ & $1.74(1.20-2.52)$ & 0.003 \\
\hline Hypercholesterolaemia, n (\%) & $306(65 \%)$ & $232(66 \%)$ & $74(63 \%)$ & $0.87(0.60-1.26)$ & 0.46 \\
\hline Hypertension, n (\%) & $397(85 \%)$ & $300(85 \%)$ & $97(82 \%)$ & $0.80(0.50-1.29)$ & 0.37 \\
\hline Current smoker, n (\%) & $39(9 \%)$ & $28(9 \%)$ & $11(10 \%)$ & $1.08(0.58-2.01)$ & 0.81 \\
\hline Coronary artery disease, $\mathrm{n}(\%)$ & $305(65 \%)$ & $221(63 \%)$ & $84(71 \%)$ & $1.36(0.91-2.02)$ & 0.13 \\
\hline Multivessel disease, n (\%) & $192(41 \%)$ & $135(38 \%)$ & $57(48 \%)$ & $1.39(0.97-1.99)$ & 0.074 \\
\hline Prior MI, n (\%) & $67(14 \%)$ & $41(12 \%)$ & $26(22 \%)$ & $1.83(1.19-2.83)$ & 0.006 \\
\hline Prior CABG, n (\%) & $37(9 \%)$ & $28(9 \%)$ & $9(9 \%)$ & $0.97(0.49-1.92)$ & 0.93 \\
\hline Prior PCI, n (\%) & $127(27 \%)$ & $92(26 \%)$ & $35(30 \%)$ & $1.16(0.78-1.71)$ & 0.48 \\
\hline Prior cerebrovascular accident, n (\%) & $52(11 \%)$ & $37(11 \%)$ & $15(13 \%)$ & $1.25(0.73-2.15)$ & 0.42 \\
\hline Peripheral artery disease, $\mathrm{n}(\%)$ & $96(20 \%)$ & $64(18 \%)$ & $32(27 \%)$ & $1.53(1.02-2.29)$ & 0.040 \\
\hline Chronic obstructive pulmonary disease, n (\%) & $79(17 \%)$ & $55(16 \%)$ & $24(20 \%)$ & $1.34(0.86-2.10)$ & 0.20 \\
\hline Renal failure (GFR <60 ml/min/1.73 $\mathrm{m}^{2}$ ) & $326(70 \%)$ & $241(69 \%)$ & $85(73 \%)$ & $1.20(0.80-1.80)$ & 0.39 \\
\hline Atrial fibrillation & $320(68 \%)$ & $248(71 \%)$ & $72(61 \%)$ & $1.46(1.01-2.11)$ & 0.045 \\
\hline Syncope, n (\%) & $66(14 \%)$ & $49(14 \%)$ & $17(14 \%)$ & $1.02(0.61-1.70)$ & 0.96 \\
\hline NYHA functional Class III/IV, n (\%) & $325(69 \%)$ & $238(68 \%)$ & $87(74 \%)$ & $1.28(0.85-1.92)$ & 0.24 \\
\hline CCS Class III/IV, n (\%) & $66(14 \%)$ & $50(14 \%)$ & $16(14 \%)$ & $0.95(0.56-1.62)$ & 0.86 \\
\hline Logistic EuroSCORE (\%) & $23.7 \pm 13.9$ & $22.4 \pm 13.1$ & $27.6 \pm 15.5$ & $1.02(1.01-1.03)$ & $<0.001$ \\
\hline STS score $(\%)$ & $6.9 \pm 4.5$ & $6.4 \pm 4.1$ & $8.4 \pm 5.4$ & 1.06 (1.04-1.09) & $<0.001$ \\
\hline \multicolumn{6}{|l|}{ Medication at admission } \\
\hline Aspirin, n (\%) & $297(64 \%)$ & $222(64 \%)$ & $75(64 \%)$ & $1.01(0.69-1.47)$ & 0.96 \\
\hline Clopidogrel, n (\%) & $91(20 \%)$ & $70(20 \%)$ & $21(18 \%)$ & $0.90(0.56-1.44)$ & 0.66 \\
\hline Oral anticoagulation, n (\%) & $126(27 \%)$ & $88(25 \%)$ & $38(32 \%)$ & $1.35(0.92-1.99)$ & 0.13 \\
\hline Diuretic, n (\%) & $334(72 \%)$ & $242(69 \%)$ & $92(79 \%)$ & $1.52(0.98-2.37)$ & 0.061 \\
\hline Beta-blocker, n (\%) & $247(53 \%)$ & $190(54 \%)$ & 57 (49\%) & $0.83(0.58-1.19)$ & 0.303 \\
\hline ACEi/ARB, n (\%) & $249(53 \%)$ & $188(54 \%)$ & $61(52 \%)$ & $0.93(0.65-1.34)$ & 0.71 \\
\hline Ca channel blocker, n (\%) & $79(17 \%)$ & 65 (19\%) & $14(12 \%)$ & $0.62(0.35-1.08)$ & 0.093 \\
\hline Statin, n (\%) & $231(50 \%)$ & $175(50 \%)$ & $56(48 \%)$ & $0.91(0.63-1.31)$ & 0.62 \\
\hline \multicolumn{6}{|c|}{$\begin{array}{l}\text { Data are expressed as mean } \pm \text { SD or number }(\%) \text {. Univariable hazard ratios }(95 \% \mathrm{Cls} \text { ) and } p \text {-values were derived from Cox regression models after } \\
\text { multiple imputation of missing covariate data (combined estimate of } 10 \text { data sets using Rubin's rule). ACE: angiotensin-converting enzyme; } \\
\text { ARB: angiotensin receptor blockers; CABG: coronary artery bypass graft; CCS: Canadian Cardiovascular Society; MI: myocardial infarction; NYHA: New } \\
\text { York Heart Association; PCI: percutaneous coronary intervention }\end{array}$} \\
\hline
\end{tabular}


Table 2. Baseline echocardiographic parameters.

\begin{tabular}{|c|c|c|c|c|c|}
\hline & $\begin{array}{l}\text { Overall } \\
n=469\end{array}$ & $\begin{array}{c}\text { Patients alive } \\
\text { at } 2 \text { years } \\
n=351\end{array}$ & $\begin{array}{c}\text { Patients dead } \\
\text { at } 2 \text { years } \\
n=118\end{array}$ & $\begin{array}{c}\text { Hazard ratio } \\
\text { (95\% CI) }\end{array}$ & $p$-value \\
\hline Aortic valve area $\left(\mathrm{cm}^{2}\right)$ & $0.7 \pm 0.2$ & $0.7 \pm 0.2$ & $0.7 \pm 0.2$ & $0.86(0.37-1.98)$ & 0.72 \\
\hline Indexed aortic valve area $\left(\mathrm{cm}^{2} \cdot \mathrm{m}^{-2}\right)$ & $0.3 \pm 0.1$ & $0.3 \pm 0.1$ & $0.3 \pm 0.1$ & $0.63(0.06-6.25)$ & 0.70 \\
\hline Aortic maximal velocity $(\mathrm{cm} / \mathrm{s})$ & $4.1 \pm 0.8$ & $4.1 \pm 0.8$ & $4.0 \pm 0.9$ & $0.87(0.67-1.13)$ & 0.30 \\
\hline Mean gradient $(\mathrm{mmHg})$ & $44.0 \pm 16.9$ & $44.3 \pm 16.1$ & $43.2 \pm 19.2$ & $0.97(0.87-1.09)^{*}$ & 0.60 \\
\hline Peak gradient $(\mathrm{mmHg})$ & $70.3 \pm 26.1$ & $70.9 \pm 24.5$ & $68.5 \pm 30.3$ & $0.97(0.90-1.05)^{*}$ & 0.43 \\
\hline LV end-diastolic diameter (mm) & $49.2 \pm 8.8$ & $48.8 \pm 8.9$ & $50.1 \pm 8.3$ & $1.14(0.90-1.43)^{*}$ & 0.28 \\
\hline LV end-systolic diameter ( $\mathrm{mm})$ & $33.6 \pm 10.4$ & $33.1 \pm 10.4$ & $35.1 \pm 10.3$ & $1.16(0.94-1.43)^{*}$ & 0.18 \\
\hline LV mass index $\left(\mathrm{g} / \mathrm{m}^{2}\right)$ & $145.7 \pm 44.3$ & $144.0 \pm 44.9$ & $150.9 \pm 42.5$ & $1.03(0.98-1.08)^{*}$ & 0.20 \\
\hline LV ejection fraction (\%) & $53.9 \pm 14.4$ & $55.1 \pm 13.8$ & $50.4 \pm 15.6$ & $0.83(0.74-0.93)^{*}$ & 0.002 \\
\hline Stroke volume index $\left(\mathrm{mL} \cdot \mathrm{m}^{-2}\right)$ & $36.8 \pm 11.8$ & $38.1 \pm 11.7$ & $33.6 \pm 11.8$ & $0.74(0.58-0.95)^{*}$ & 0.019 \\
\hline TAPSE (mm) & $18.0 \pm 5.1$ & $18.2 \pm 4.8$ & $17.7 \pm 5.8$ & $0.98(0.93-1.03)$ & 0.43 \\
\hline DTI velocity $\left(\mathrm{cm} \cdot \mathrm{sec}^{-1}\right)$ & $11.5 \pm 3.1$ & $11.3 \pm 3.0$ & $11.9 \pm 3.2$ & $1.04(0.96-1.13)$ & 0.31 \\
\hline Aortic regurgitation moderate or severe & $36(8 \%)$ & $29(9 \%)$ & $7(6 \%)$ & $0.80(0.37-1.71)$ & 0.57 \\
\hline Mitral regurgitation moderate or severe & $119(25 \%)$ & $74(21 \%)$ & $45(38 \%)$ & $2.04(1.41-2.96)$ & $<0.001$ \\
\hline Tricuspid regurgitation moderate or severe & $89(19 \%)$ & $55(16 \%)$ & $34(29 \%)$ & $1.94(1.30-2.89)$ & 0.001 \\
\hline RV-RA gradient $(\mathrm{mmHg})$ & $39.2 \pm 12.5$ & $37.4 \pm 12.3$ & $44.8 \pm 11.2$ & $1.04(1.02-1.05)$ & $<0.001$ \\
\hline Central venous pressure $(\mathrm{mmHg})$ & $8.9 \pm 3.8$ & $8.5 \pm 3.7$ & $10.2 \pm 3.8$ & $1.10(1.04-1.16)$ & $<0.001$ \\
\hline Pulmonary artery systolic pressure $(\mathrm{mmHg})$ & $48.3 \pm 14.3$ & $46.1 \pm 14.1$ & $55.3 \pm 12.7$ & $1.04(1.02-1.05)$ & $<0.001$ \\
\hline
\end{tabular}

$55.1 \pm 13.8 \%$, HR 0.83, 95\% CI: 0.74-0.93, $\mathrm{p}=0.002$ ), moderate or severe mitral regurgitation (38\% vs. $21 \%$, HR $2.04,95 \%$ CI: $1.41-2.96, \mathrm{p}<0.001)$ and moderate or severe tricuspid regurgitation (29\% vs. $16 \%$, HR 1.94, 95\% CI: $1.30-2.89$, p<0.001) were associated with an increased risk of death at two years.

\section{HAEMODYNAMIC PARAMETERS AT PREOPERATIVE RHC}

RHC-derived measures are reported in Table 3. Patients who had died within two years after intervention were more likely to have higher mean PAP $(35.09 \pm 10.58$ vs. $32.51 \pm 11.88 \mathrm{mmHg}$, HR 1.19, 95\% CI: 1.02-1.38, p=0.029), lower pulmonary arterial compliance $\left(1.56 \pm 0.81\right.$ vs. $1.89 \pm 0.95 \mathrm{ml} . \mathrm{mmHg}^{-1}$, HR 0.65 , 95\% CI: 0.51-0.84, $\mathrm{p}=0.001)$, higher transpulmonic gradient (14.22 \pm 10.41 vs. $10.95 \pm 11.44$, HR $1.25,95 \%$ CI: 1.07-1.47, $\mathrm{p}=0.006)$, higher pulmonary vascular resistance $(359.84 \pm 308.99$ vs. 265.46 $\pm 302.50 \mathrm{mmHg}$.min. $\mathrm{L}^{-1}$, HR 1.09, 95\% CI: 1.03-1.15, $\mathrm{p}=0.003)$ and higher mean RAP $(8.97 \pm 6.13$ vs. $7.76 \pm 4.55 \mathrm{mmHg}$, HR 1.61, 95\% CI: 1.13-2.31, $\mathrm{p}=0.009)$.

\section{INDEPENDENT PREDICTORS OF DEATH AT TWO YEARS}

Relevant variables among clinical, echocardiographic and haemodynamic measures were entered in a multivariable Cox proportional analysis in a forward stepwise fashion (Table 4): diabetes (HR 1.95, 95\% CI: 1.28-2.96, p=0.001), transapical access (HR 1.66, 95\% CI: $1.07-2.56, \mathrm{p}=0.02$ ) and moderate or severe mitral regurgitation (HR 1.55, 95\% CI: 1.00-2.39, p=0.04) at baseline were independently associated with all-cause mortality at two years.

\section{LACK OF INCREMENTAL UTILITY OF RHC TO PREDICT MORTALITY AFTER TAVI}

Sequential models for the prediction of death were created to assess the incremental contribution of preoperative RHC relative to clinical and echocardiographic parameters. As shown in Figure 1, the Harrell's C-statistic was 0.662 (95\% CI: $0.612-$ 0.713 ) in a model including clinical and echocardiographic parameters and 0.667 (95\% CI: 0.615-0.719) with implementation of RHC-derived metrics $(\mathrm{p}=0.47)$.

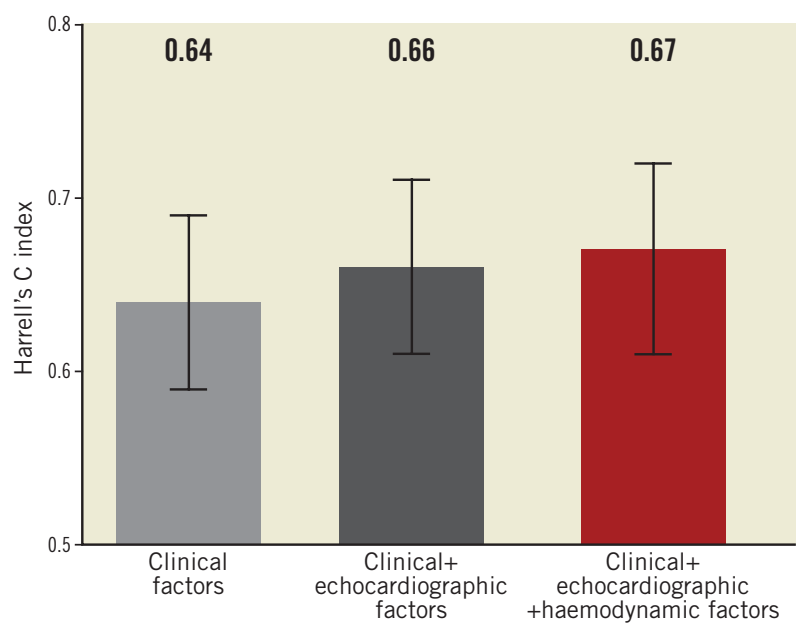

Figure 1. RHC prior to TAVI does not increase the prognostic value of clinical and echocardiographic factors. 
Table 3. Measures at preoperative RHC.

\begin{tabular}{|c|c|c|c|c|c|}
\hline & $\begin{array}{l}\text { Overall } \\
n=469\end{array}$ & $\begin{array}{c}\text { Patients alive } \\
\text { at } 2 \text { years } \\
n=351\end{array}$ & $\begin{array}{l}\text { Patients dead } \\
\text { at } 2 \text { years } \\
n=118\end{array}$ & $\begin{array}{c}\text { Hazard ratio } \\
\text { (95\% CI) }\end{array}$ & p-value \\
\hline Systolic arterial pressure $(\mathrm{mmHg})$ & $136 \pm 28.4$ & $137.4 \pm 28.5$ & $131.7 \pm 27.7$ & $0.94(0.88-1.00)$ & 0.056 \\
\hline Diastolic arterial pressure $(\mathrm{mmHg})$ & $65.7 \pm 13.9$ & $66.3 \pm 14.1$ & $63.9 \pm 12.9$ & $0.90(0.79-1.03)$ & 0.12 \\
\hline Mean arterial pressure $(\mathrm{mmHg})$ & $93.9 \pm 17.8$ & $94.7 \pm 18.0$ & $91.3 \pm 16.7$ & $0.91(0.82-1.01)$ & 0.081 \\
\hline Systemic vascular resistance $\left(\right.$ dynes $\cdot \mathrm{cm}^{-5}$ ) & $1,964.1 \pm 670.1$ & $1,954.5 \pm 648.1$ & $1,993.1 \pm 734.9$ & $1.01(0.98-1.04)$ & 0.55 \\
\hline Systemic arterial compliance $\left(\mathrm{ml} \cdot \mathrm{mmHg}^{-1}\right)$ & $0.44 \pm 0.20$ & $0.43 \pm 0.18$ & $0.45 \pm 0.24$ & $1.62(0.64-4.12)$ & 0.31 \\
\hline Valvulo-arterial impedance $\left(\mathrm{mmHg} \cdot \mathrm{ml} \cdot \mathrm{m}^{2}\right)$ & $7.34 \pm 2.57$ & $7.33 \pm 2.52$ & $7.39 \pm 2.71$ & $1.01(0.94-1.09)$ & 0.74 \\
\hline LV systolic pressure $(\mathrm{mmHg})$ & $188.2 \pm 34.7$ & $190.6 \pm 33.1$ & $180.8 \pm 38.4$ & $0.93(0.88-0.98)$ & 0.011 \\
\hline LV end-diastolic pressure (mmHg) & $21.4 \pm 8.0$ & $21.5 \pm 8.1$ & $20.1 \pm 7.7$ & $0.93(0.73-1.17)$ & 0.51 \\
\hline LV development pressure $(\mathrm{mmHg})$ & $166.7 \pm 32.8$ & $169.0 \pm 30.9$ & $159.8 \pm 37.2$ & $0.93(0.87-0.98)$ & 0.010 \\
\hline LV stroke work $(\mathrm{g} \cdot \mathrm{m})$ & $92.0 \pm 32.6$ & $94.2 \pm 31.9$ & $85.2 \pm 33.9$ & $0.92(0.86-0.98)$ & 0.012 \\
\hline Stroke volume (ml) & $49.5 \pm 15.7$ & $50.2 \pm 15.6$ & $47.2 \pm 15.9$ & $0.89(0.78-1.01)$ & 0.061 \\
\hline Stroke volume index $\left(\mathrm{ml} / \mathrm{m}^{2}\right)$ & $27.7 \pm 7.9$ & $28.1 \pm 8.0$ & $26.6 \pm 7.8$ & $0.80(0.63-1.02)$ & 0.072 \\
\hline Cardiac output (L/min) & $3.7 \pm 1.0$ & $3.8 \pm 0.9$ & $3.6 \pm 1.1$ & $0.84(0.69-1.02)$ & 0.072 \\
\hline Cardiac index $\left(\mathrm{L} / \mathrm{min} / \mathrm{m}^{2}\right)$ & $2.1 \pm 0.5$ & $2.1 \pm 0.5$ & $2.0 \pm 0.5$ & $0.71(0.48-1.03)$ & 0.074 \\
\hline PA systolic pressure $(\mathrm{mmHg})$ & $51.6 \pm 17.1$ & $50.2 \pm 17.1$ & $55.5 \pm 16.5$ & $1.16(1.05-1.28)$ & 0.003 \\
\hline PA diastolic pressure $(\mathrm{mmHg})$ & $20.2 \pm 8.7$ & $19.8 \pm 8.8$ & $21.4 \pm 8.0$ & $1.22(0.99-1.50)$ & 0.061 \\
\hline Mean PA pressure $(\mathrm{mmHg})$ & $33.2 \pm 11.6$ & $32.5 \pm 11.9$ & $35.1 \pm 10.6$ & $1.19(1.02-1.38)$ & 0.029 \\
\hline Pulmonary arterial compliance $\left(\mathrm{ml} \cdot \mathrm{mmHg}^{-1}\right)$ & $1.8 \pm 0.9$ & $1.9 \pm 0.9$ & $1.5 \pm 0.8$ & $0.65(0.51-0.84)$ & 0.001 \\
\hline Diastolic pulmonary gradient $(\mathrm{mmHg})$ & $-1.1 \pm 9.0$ & $-1.7 \pm 9.1$ & $0.6 \pm 8.6$ & $1.03(1.01-1.05)$ & 0.013 \\
\hline Transpulmonic gradient (mmHg) & $11.7 \pm 11.2$ & $10.9 \pm 11.4$ & $14.2 \pm 10.4$ & $1.25(1.07-1.47)$ & 0.006 \\
\hline Pulmonary vascular resistance $\left(\mathrm{mmHg} \cdot \mathrm{min} \cdot \mathrm{L}^{-1}\right)$ & $289.11 \pm 306.54$ & $265.4 \pm 302.5$ & $359.8 \pm 309$ & $1.09(1.03-1.15)$ & 0.003 \\
\hline Pulmonary vascular resistance (Wood units) & $3.6 \pm 3.9$ & $3.3 \pm 3.8$ & $4.5 \pm 3.9$ & $1.07(1.02-1.12)$ & 0.003 \\
\hline RV systolic pressure (mmHg) & $51 \pm 16.5$ & $49.9 \pm 16.4$ & $54.3 \pm 16.3$ & $1.15(1.04-1.27)$ & 0.009 \\
\hline RV end-diastolic pressure $(\mathrm{mmHg})$ & $9.9 \pm 5.4$ & $9.9 \pm 5.3$ & $10.2 \pm 5.8$ & $1.12(0.80-1.58)$ & 0.50 \\
\hline RA mean pressure $(\mathrm{mmHg})$ & $8.1 \pm 5.0$ & $7.8 \pm 4.5$ & $9 \pm 6.1$ & $1.61(1.13-2.31)$ & 0.009 \\
\hline RV stroke work index $\left(\mathrm{g} \cdot \mathrm{m} \cdot \mathrm{m}^{-2} \cdot\right.$ beat $\left.^{-1}\right)$ & $32.3 \pm 11.1$ & $33.2 \pm 11.3$ & $29.4 \pm 10.2$ & $0.97(0.95-0.99)$ & 0.001 \\
\hline Aortic saturation (\%) & $94 \pm 3.6$ & $94 \pm 3.6$ & $94.1 \pm 3.6$ & $1.07(0.64-1.78)$ & 0.80 \\
\hline Pulmonary artery saturation (\%) & $60.8 \pm 8.5$ & $61.6 \pm 8.3$ & $58.4 \pm 8.9$ & $0.71(0.58-0.85)$ & $<0.001$ \\
\hline
\end{tabular}

\section{ROLE OF RHC AMONG PATIENTS WITH PULMONARY HYPERTENSION AT BASELINE}

Among patients with baseline pulmonary hypertension, defined as mean PAP $\geq 25 \mathrm{mmHg}$, clinical and echocardiographic variables (age: HR 1.06, 95\% CI: 1.01-1.12, $\mathrm{p}=0.03$; diabetes: HR 2.46, 95\% CI: $1.52-3.96, \mathrm{p}<0.001$; moderate or severe mitral regurgitation: HR 1.67, 95\% CI: 1.03-2.70, p=0.03), but not haemodynamic indices, were predictors of two-year mortality. The addition of haemodynamic variables did not significantly increase the prognostic value of a model including clinical and echocardiographic parameters (Figure 2).

\section{Discussion}

In the present study, RHC-derived measures failed to predict mortality among patients undergoing TAVI; furthermore, the addition of haemodynamic parameters did not significantly improve the prognostic power of a model containing clinical and echocardiographic variables. Intracardiac haemodynamics featuring patients
Table 4. Multivariable model.

\begin{tabular}{|l|c|l|}
\hline Age (years) & $\begin{array}{c}\text { Hazard ratio } \\
(95 \% \text { CI) }\end{array}$ & $\boldsymbol{p}$-value \\
\hline Body mass index $\left(\mathrm{kg} / \mathrm{m}^{2}\right)$ & $1.04(1.00-1.08)$ & 0.082 \\
\hline Diabetes mellitus & $0.98(0.94-1.02)$ & 0.32 \\
\hline Previous cerebrovascular accident & $1.95(1.28-2.96)$ & 0.001 \\
\hline Chronic obstructive pulmonary disease & $1.30(0.75-2.26)$ & 0.36 \\
\hline Transapical access & $1.41(0.87-2.27)$ & 0.17 \\
\hline STS risk score & $1.66(1.07-2.56)$ & 0.02 \\
\hline Mitral regurgitation (moderate or severe) & $1.55(1.00-2.39)$ & 0.047 \\
\hline Left ventricular ejection fraction & $0.91(0.79-1.05)$ & 0.20 \\
\hline Mean aortic valve gradient & $1.05(0.92-1.18)$ & 0.49 \\
\hline $\begin{array}{l}\text { Pulmonary arterial compliance } \\
\left.\text { (ml-mmHg }{ }^{-1}\right)\end{array}$ & $0.73(0.47-1.13)$ & 0.16 \\
\hline RA mean pressure (mmHg) & $1.13(0.75-1.69)$ & 0.56 \\
\hline $\begin{array}{l}\text { Multivariable model after multiple imputation of missing values, } \\
\text { estimates from combining } 20 \text { imputed data sets. RA: right atrium }\end{array}$ \\
\hline
\end{tabular}




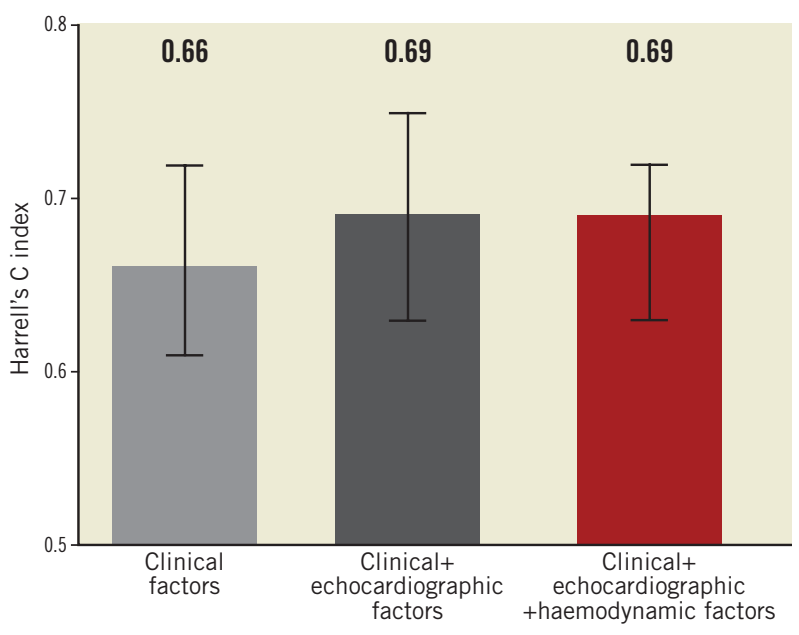

Figure 2. RHC prior to TAVI does not increase the prognostic value of clinical and echocardiographic factors among patients with pulmonary hypertension at baseline.

with severe AS have been previously assessed ${ }^{9}$. The haemodynamic parameter with the highest prognostic value in the setting of severe AS is undoubtedly pulmonary hypertension. It operates the continuum between left ventricular dysfunction and right heart failure in the context of pressure overload: indeed, the backward transmission of the elevated pressures in the hypertrophic left ventricle promotes the remodelling of pulmonary vasculature. The presence of pulmonary hypertension affects the prognosis of patients with severe AS irrespective of the treatment ${ }^{7,10,11}$. In this context, we sought to investigate whether other invasive haemodynamic parameters, among those mirroring the pathophysiology of the valvular disease, could be related to mortality after TAVI.

The prognostic significance of several RHC-derived measures has been assessed in other clinical settings. Specifically, elevated mean right atrial pressure and lower cardiac index independently predict mortality among patients with pulmonary arterial hypertension (group I) ${ }^{1}$. A reduced pulmonary arterial compliance proved a powerful marker of poor prognosis in decompensated heart failure ${ }^{12}$. Nevertheless, in our study, none of the haemodynamic parameters obtained at RHC prior to TAVI proved to have better capability to predict mortality than clinical factors. Among these, diabetes is quoted within the scores commonly used to estimate the operative risk of patients. Analyses of registry data showed that insulin-treated subjects and those with poorer glycaemic control (as indicated by higher values of glycated haemoglobin A1c) were at increased risk for mortality at long-term follow-up ${ }^{13,14}$. Indeed, diabetic status is supposed to impair the left ventricular reverse remodelling and regression of hypertrophy triggered by the relief of valvular stenosis ${ }^{15}$. Similarly, the independent association between moderate or severe mitral regurgitation at baseline and the risk of death after TAVI has been widely documented ${ }^{16-18}$. In addition, the survival advantage of transfemoral compared with transapical TAVI is also in line with previous findings. A significant survival benefit in the subgroup of patients allocated to transfemoral TAVI compared with surgical intervention has been reported in a recent meta-analysis of randomised trials including patients at high risk and lower-than-high risk ${ }^{19}$. Patient- and procedure-related factors contribute to enhance the risk of worse outcomes among subjects undergoing transapical TAVI. The patients are typically older and have more cardiovascular risk factors. In addition, access-related myocardial damage can affect the recovery of left ventricular function and provide the substrate for arrhythmic events.

In our study, the superior prognostic value of clinical over haemodynamic factors was confirmed in the subgroup of patients with pulmonary hypertension at baseline.

Similarly, in a sub-analysis of the PARTNER-I trial, including patients with preoperative moderate or severe pulmonary hypertension, clinical factors such as oxygen-dependent lung disease, inability to perform a six-minute walk test, impaired renal function and lower aortic valve mean gradient proved more informative about survival at one year compared with RHC-derived measures ${ }^{20}$. Yet, while haemodynamic variables, such as the mean right atrial pressure, proved to have independent prognostic ability in patients with pulmonary arterial hypertension (group I), they failed to predict mortality in subjects with pulmonary hypertension secondary to left heart diseases (group II) ${ }^{21}$.

Overall, our results suggest that clinical factors affect long-term outcomes after TAVI to a greater extent than haemodynamic parameters. In this regard, clinical characteristics and comorbidities at baseline represent the variables with greatest prognostic weight in recently developed scores to predict early and late mortality after TAVI ${ }^{22}$.

Previous investigations by our group focused on the effect of haemodynamic patterns of pulmonary hypertension or low-flow, low-gradient aortic stenosis on clinical outcomes after TAVI ${ }^{7,23}$. Instead, the present analysis sought to determine whether preoperative RHC provides additional prognostic value on top of standard, non-invasive evaluation including clinical and echocardiographic parameters. Although several haemodynamic parameters were associated with two-year mortality at univariable analysis, there was a lack of incremental prognostic information provided by RHC when clinical and echocardiographic parameters were considered. The present study, therefore, provides a nuanced appreciation of the role of invasive haemodynamic measurements before TAVI and suggests that RHC could be a dispensable tool for prognostication in the preoperative diagnostic workup if comprehensive non-invasive information is already available.

\section{Limitations}

We acknowledge the following limitations of our analysis: 1) the retrospective design of the study conveys intrinsic biases such as the unrecognised presence of confounding factors with impact on mortality after TAVI; 2) referral bias can be present as patients received RHC prior to TAVI on the basis of their clinical history; in particular, haemodynamically unstable patients may have been referred to TAVI without previous RHC; 3) we did not assess 
whether changes in haemodynamic intracardiac profile occurred after TAVI and whether they had an impact on long-term mortality; 4) as the study cohort comprised elderly and high-risk patients, the results could not apply to younger and lower-risk subjects; 5) while complications resulting from preoperative left and right heart catheterisation were generally rare and limited to access-site complications, adverse events attributable to RHC were not systematically collected in the setting of our study.

\section{Conclusions}

The invasive assessment of haemodynamic parameters provides important information on the pathophysiology of aortic stenosis, patient prognosis, and functional status after valve replacement. Nevertheless, in an era witnessing a progressive streamlining of TAVI procedures, routine RHC may become a dispensable tool for prognosis assessment when comprehensive data from non-invasive diagnostics are available.

\section{Impact on daily practice}

This study suggests that clinical baseline characteristics have a greater prognostic impact than haemodynamic indices in patients evaluated for transcatheter aortic valve implantation (TAVI).

\section{Conflict of interest statement}

$\mathrm{S}$. Windecker reports having received research grants to the institution from Abbott, Biotronik, Boston Scientific, Edwards Lifesciences, Medtronic, The Medicines Company, and St. Jude as well as speaker fees from AstraZeneca, Eli Lilly, Abbott, Biotronik, Boston Scientific, Bayer, and Biosensors. L. Räber has received speaker fees and research grants to the institution from St. Jude Medical. The other authors have no conflicts of interest to declare.

\section{References}

1. Benza RL, Miller DP, Gomberg-Maitland M, Frantz RP, Foreman AJ, Coffey CS, Frost A, Barst RJ, Badesch DB, Elliott CG, Liou TG, McGoon MD. Predicting survival in pulmonary arterial hypertension: insights from the Registry to Evaluate Early and Long-Term Pulmonary Arterial Hypertension Disease Management (REVEAL). Circulation. 2010;122:164-72.

2. Pellegrini P, Rossi A, Pasotti M, Raineri C, Cicoira M, Bonapace S, Dini FL, Temporelli PL, Vassanelli C, Vanderpool R, Naeije R, Ghio S. Prognostic relevance of pulmonary arterial compliance in patients with chronic heart failure. Chest. 2014;145:1064-70.

3. Pibarot P, Dumesnil JG. New concepts in valvular hemodynamics: implications for diagnosis and treatment of aortic stenosis. Can J Cardiol. 2007;23 Suppl B:40B-47B.

4. Kupari M, Turto H, Lommi J. Left ventricular hypertrophy in aortic valve stenosis: preventive or promotive of systolic dysfunction and heart failure? Eur Heart J. 2005;26:1790-6.

5. Lucon A, Oger E, Bedossa M, Boulmier D, Verhoye JP, Eltchaninoff H, Iung B, Leguerrier A, Laskar M, Leprince P,
Gilard M, Le Breton H. Prognostic implications of pulmonary hypertension in patients with severe aortic stenosis undergoing transcatheter aortic valve implantation: study from the FRANCE 2 Registry. Circ Cardiovasc Interv. 2014;7:240-7.

6. Ben-Dor I, Goldstein SA, Pichard AD, Satler LF, Maluenda G, Li Y, Syed AI, Gonzalez MA, Gaglia MA Jr, Wakabayashi K, Delhaye C, Belle L, Wang Z, Collins SD, Torguson R, Okubagzi P, Aderotoye A, Xue Z, Suddath WO, Kent KM, Epstein SE, Lindsay J, Waksman R. Clinical profile, prognostic implication, and response to treatment of pulmonary hypertension in patients with severe aortic stenosis. Am J Cardiol. 2011;107:1046-51.

7. O'Sullivan CJ, Wenaweser P, Ceylan O, Rat-Wirtzler J, Stortecky S, Heg D, Spitzer E, Zanchin T, Praz F, Tuller D, Huber C, Pilgrim T, Nietlispach F, Khattab AA, Carrel T, Meier B, Windecker S, Buellesfeld L. Effect of Pulmonary Hypertension Hemodynamic Presentation on Clinical Outcomes in Patients With Severe Symptomatic Aortic Valve Stenosis Undergoing Transcatheter Aortic Valve Implantation: Insights From the New Proposed Pulmonary Hypertension Classification. Circ Cardiovasc Interv. 2015;8:e02358.

8. Wenaweser P, Pilgrim T, Kadner A, Huber C, Stortecky S, Buellesfeld L, Khattab AA, Meuli F, Roth N, Eberle B, Erdos G, Brinks H, Kalesan B, Meier B, Jüni P, Carrel T, Windecker S. Clinical outcomes of patients with severe aortic stenosis at increased surgical risk according to treatment modality. $\mathrm{J} \mathrm{Am} \mathrm{Coll} \mathrm{Cardiol.}$ 2011;58:2151-62.

9. Chang SA, Park PW, Sung K, Lee SC, Park SW, Lee YT, Oh JK. Noninvasive estimate of left ventricular filling pressure correlated with early and midterm postoperative cardiovascular events after isolated aortic valve replacement in patients with severe aortic stenosis. J Thorac Cardiovasc Surg. 2010;140:1361-6.

10. Zlotnick DM, Ouellette ML, Malenka DJ, DeSimone JP, Leavitt BJ, Helm RE, Olmstead EM, Costa SP, DiScipio AW, Likosky DS, Schmoker JD, Quinn RD, Sisto D, Klemperer JD, Sardella GL, Baribeau YR, Frumiento C, Brown JR, O'Rourke DJ; Northern New England Cardiovascular Disease Study Group. Effect of preoperative pulmonary hypertension on outcomes in patients with severe aortic stenosis following surgical aortic valve replacement. Am J Cardiol. 2013;112:1635-40.

11. Pilgrim T, Englberger L, Rothenbühler M, Stortecky S, Ceylan O, O'Sullivan CJ, Huber C, Praz F, Buellesfeld L, Langhammer B, Meier B, Jüni P, Carrel T, Windecker S, Wenaweser P. Long-term outcome of elderly patients with severe aortic stenosis as a function of treatment modality. Heart. 2015;101:30-6.

12. Miller WL, Grill DE, Borlaug BA. Clinical features, hemodynamics, and outcomes of pulmonary hypertension due to chronic heart failure with reduced ejection fraction: pulmonary hypertension and heart failure. JACC Heart Fail. 2013;1:290-9.

13. Abramowitz Y, Jilaihawi H, Chakravarty $T$, Mangat G, Maeno Y, Kazuno Y, Takahashi N, Kawamori H, Cheng W, Makkar RR. Impact of Diabetes Mellitus on Outcomes After Transcatheter Aortic Valve Implantation. Am J Cardiol. 2016;117: $1636-42$. 
14. Chorin E, Finkelstein A, Banai S, Aviram G, Barkagan M, Barak L, Keren G, Steinvil A. Impact of Diabetes Mellitus and Hemoglobin A1C on Outcome After Transcatheter Aortic Valve Implantation. Am J Cardiol. 2015;116:1898-903.

15. Nakamura T, Toda K, Kuratani T, Miyagawa S, Yoshikawa Y, Fukushima S, Saito S, Yoshioka D, Kashiyama N, Daimon T, Sawa Y. Diabetes Mellitus Impairs Left Ventricular Mass Regression after Surgical or Transcatheter Aortic Valve Replacement for Severe Aortic Stenosis. Heart Lung Circ. 2016;25:68-74.

16. Takagi $\mathrm{H}$, Umemoto $\mathrm{T}$; All-Literature Investigation of Cardiovascular Evidence Group. Coexisting Mitral Regurgitation Impairs Survival After Transcatheter Aortic Valve Implantation. Ann Thorac Surg. 2015;100:2270-6.

17. Khawaja MZ, Williams R, Hung J, Arri S, Asrress KN, Bolter K, Wilson K, Young CP, Bapat V, Hancock J, Thomas M, Redwood S. Impact of preprocedural mitral regurgitation upon mortality after transcatheter aortic valve implantation (TAVI) for severe aortic stenosis. Heart. 2014;100:1799-803.

18. Sannino A, Losi MA, Schiattarella GG, Gargiulo G, Perrino C, Stabile E, Toscano E, Giugliano G, Brevetti L, Franzone A, Cirillo P, Imbriaco M, Trimarco B, Esposito G. Meta-analysis of mortality outcomes and mitral regurgitation evolution in 4,839 patients having transcatheter aortic valve implantation for severe aortic stenosis. Am J Cardiol. 2014;114:875-82.

19. Siontis GC, Praz F, Pilgrim T, Mavridis D, Verma S, Salanti G, Sondergaard L, Jüni P, Windecker S. Transcatheter aortic valve implantation vs. surgical aortic valve replacement for treatment of severe aortic stenosis: a meta-analysis of randomized trials. Eur Heart J. 2016;37:3503-3512.

20. Lindman BR, Zajarias A, Maniar HS, Miller DC, Suri RM, Arnold SV, Webb J, Svensson LG, Kodali S, Xu K, Ayele GM, Lin F, Wong SC, Babaliaros V, Thourani VH, Douglas PS, Lim S, Leon MB, Mack MJ. Risk stratification in patients with pulmonary hypertension undergoing transcatheter aortic valve replacement. Heart. 2015;101:1656-64.

21. Yamabe S, Dohi Y, Fujisaki S, Higashi A, Kinoshita H, Sada Y, Hidaka T, Kurisu S, Yamamoto H, Kihara Y. Prognostic Factors for Survival in Pulmonary Hypertension Due to Left Heart Disease. Circ J. 2016;80:243-9.

22. Edwards FH, Cohen DJ, O'Brien SM, Peterson ED, Mack M, Shanian D, Grover FL, Tuzcu EM, Thourani VH, Carrol J, Brennan M, Brindis R, Rumsfeld J, Holmes DR Jr; Steering Committee of the Society of Thoracic Surgeons/American College of Cardiology Transcatheter Valve Therapy Registry. Development and Validation of a Risk Prediction Model for In-Hospital Mortality After Transcatheter Aortic Valve Replacement. JAMA Cardiol. 2016;1:46-52.

23. O’Sullivan CJ, Stortecky S, Heg D, Pilgrim T, Hosek N, Buellesfeld L, Khattab AA, Nietlispach F, Moschovitis A, Zanchin T, Meier B, Windecker S, Wenaweser P. Clinical outcomes of patients with low-flow, low-gradient, severe aortic stenosis and either preserved or reduced ejection fraction undergoing transcatheter aortic valve implantation. Eur Heart J. 2013;34: 3437-50. 